\title{
Research Article \\ On the Velocity of Moving Relativistic Unstable Quantum Systems
}

\author{
K. Urbanowski \\ Institute of Physics, University of Zielona Góra, Ulica Prof. Z. Szafrana 4a, 65-516 Zielona Góra, Poland \\ Correspondence should be addressed to K. Urbanowski; k.urbanowski@if.uz.zgora.pl
}

Received 10 October 2015; Revised 13 December 2015; Accepted 17 December 2015

Academic Editor: Shi-Hai Dong

Copyright ( $) 2015 \mathrm{~K}$. Urbanowski. This is an open access article distributed under the Creative Commons Attribution License, which permits unrestricted use, distribution, and reproduction in any medium, provided the original work is properly cited. The publication of this article was funded by SCOAP . $^{3}$

\begin{abstract}
We study properties of moving relativistic quantum unstable systems. We show that in contrast to the properties of classical particles and quantum stable objects the velocity of freely moving relativistic quantum unstable systems cannot be constant in time. We show that this new quantum effect results from the fundamental principles of the quantum theory and physics: it is a consequence of the principle of conservation of energy and of the fact that the mass of the quantum unstable system is not defined. This effect can affect the form of the decay law of moving relativistic quantum unstable systems.
\end{abstract}

\section{Introduction}

Physicists studying the decay processes of unstable quantum systems moving with the velocity $\vec{v}$ relative to the rest reference frame of an observer and trying to derive theoretically the decay law of such systems are confronted with the following problem: Which of the two possible assumptions, $\vec{v}=$ const or perhaps $\vec{p}=$ const (where $\vec{p}$ is the momentum of the moving unstable system), will get decay law correctly describing the real properties of such system. When one considers classical physics decay processes, the mentioned assumptions both lead to the decay law of the same form. Namely, from the standard, text book considerations, one finds that if the decay law of the unstable particle in rest has the exponential form $\mathscr{P}_{0}(t)=\exp \left[-\Gamma_{0} t\right]$, then the decay law of the moving particle with momentum $\vec{p} \neq 0$ is $\mathscr{P}_{p}(t)=$ $\mathscr{P}_{0}(t / \gamma) \equiv \exp \left[-\Gamma_{0} t / \gamma\right] \equiv \mathscr{P}_{v}(t)$, where $t$ denotes time, $\Gamma_{0}$ is the decay rate (time $t$ and $\Gamma_{0}$ are measured in the rest reference frame of the particle), $\gamma$ is the relativistic Lorentz factor, $\gamma \equiv 1 / \sqrt{1-v^{2}}, v=|\vec{v}|$, and $\mathscr{P}_{v}(t)$ is the decay law of the particle moving with the constant velocity $\vec{v}$ (we use $\hbar=c=1$ units, and thus $v<1$ ). It is almost common belief that this equality is valid also for any $t$ in the case of quantum decay processes and does not depend on the model of the unstable system considered. The cases $\vec{p}=$ const and $\vec{v}=$ const both were studied in the literature. The assumption $\vec{p}=$ const was used in $[1,2]$ to derive the survival probability $\mathscr{P}_{p}(t)$. From these studies, it follows that in the case of moving quantum unstable systems the relation $\mathscr{P}_{p}(t) \simeq \mathscr{P}_{0}(t / \gamma)$ is valid to a sufficient accuracy only for not more than a few lifetimes and that for times much longer than a few lifetimes there is $\mathscr{P}_{p}(t)>\mathscr{P}_{0}(t / \gamma)($ see $[2,3])$. The assumption $\vec{v}=$ const was used, for example, in [4], to derive the decay law of moving quantum unstable systems. Unfortunately, the result obtained in [4] is similar to the case $\vec{p}=$ const: $\mathscr{P}_{v}(t) \simeq \mathscr{P}_{0}(t / \gamma)$ only for no more than a few lifetimes. What is more, it appears that the assumption $\vec{v}=$ const may lead to the relation $\mathscr{P}_{v}(t)=$ $\mathscr{P}_{0}(\gamma t)$, that is, to the result never observed in experiments $[5,6]$.

Unfortunately, the experiments did not give any decisive answer for the problem which is the correct assumption: $\vec{p}=$ const or $\vec{v}=$ const? It is because all known tests of the relation $\mathscr{P}_{v(p)}(t) \simeq \mathscr{P}_{0}(t / \gamma)$ were performed for times $t \sim \tau_{0}$ (where $\tau_{0}$ is the lifetime) (see, e.g., [7, $8])$. Note that the same relation obtained in $[1,2,4]$ is approximately valid for the same times $t$ (see also discussion in [9-13]). The problem seems to be extremely important in accelerator physics where the correct interpretation of the obtained results depends on knowledge of the properly calculated decay law of the moving unstable particles created in 
the collisions observed. Similarly, the proper interpretation of results of observations of astrophysical processes in which a huge numbers of elementary particles (including unstable one) are produced is impossible without knowing the correct form of the decay law of unstable particles created in these processes. So the further theoretical studies of the abovedescribed problem are necessary and seem to be important.

In this paper, we analyze general properties of unstable quantum system from the point of view of fundamental principles of physics and quantum theory. Here, we show that the principle of the conservation of the energy does not allow any moving quantum unstable system to move with the velocity $\vec{v}$ constant in time.

\section{Quantum Unstable Systems}

The main information about properties of quantum unstable systems is contained in their decay law, that is, in their survival probability. Let the reference frame $\mathcal{O}$ be the common inertial rest frame for the observer and for the unstable system. Then, if one knows that the system in the rest frame is in the initial unstable state $|\phi\rangle$, which was prepared at the initial instant $t_{0}=0$, one can calculate its survival probability, $\mathscr{P}_{0}(t)$, which equals $\mathscr{P}_{0}(t)=|a(t)|^{2}$, where $a(t)$ is the survival amplitude, $a(t)=\langle\phi \mid \phi ; t\rangle,|\phi ; t\rangle=e^{-i t H}|\phi\rangle, H$ is the total self-adjoint Hamiltonian of the system under considerations, $|\phi\rangle,|\phi ; t\rangle \in \mathscr{H}$, and $\mathscr{H}$ is the Hilbert space of states of the considered system. So in order to calculate the amplitude $a(t)$, one should know the state $|\phi\rangle$. Within the standard approach, the unstable state $|\phi\rangle$ is modeled as the following wave packet [14-18]:

$$
|\phi\rangle=\int_{\mu_{0}}^{\infty} c(m)|m\rangle d m,
$$

where $\mu_{0}$ is the lower bound of the continuous part $\sigma_{c}(H)$ of the spectrum of $H$ and vectors $|m\rangle$ solve the following:

$$
H|m\rangle=m|m\rangle, \quad m \in \sigma_{c}(H) .
$$

Eigenvectors $|m\rangle$ are normalized as follows:

$$
\left\langle m \mid m^{\prime}\right\rangle=\delta\left(m-m^{\prime}\right) .
$$

We require the state $|\phi\rangle$ to be normalized; so it has to be $\int_{\mu_{0}}^{\infty}|c(m)|^{2} d m=1$. Thus,

$$
|\phi ; t\rangle=e^{-i t H}|\phi\rangle \equiv \int_{\mu_{0}}^{\infty} c(m) e^{-i t m}|m\rangle d m,
$$

which allows one to represent the amplitude $a(t)$ as the Fourier transform of the mass (energy) distribution function, $\omega(m) \equiv|c(m)|^{2}:$

$$
a(t)=\int \omega(m) e^{-i t m} d m
$$

where $\omega(m) \geq 0$ and $\omega(m)=0$, for $m<\mu_{0}$ [14-23] (see also [1-6]). From the last relation and from the RiemannLebesque lemma, it follows that $|a(t)| \rightarrow 0$ as $t \rightarrow \infty$. It is

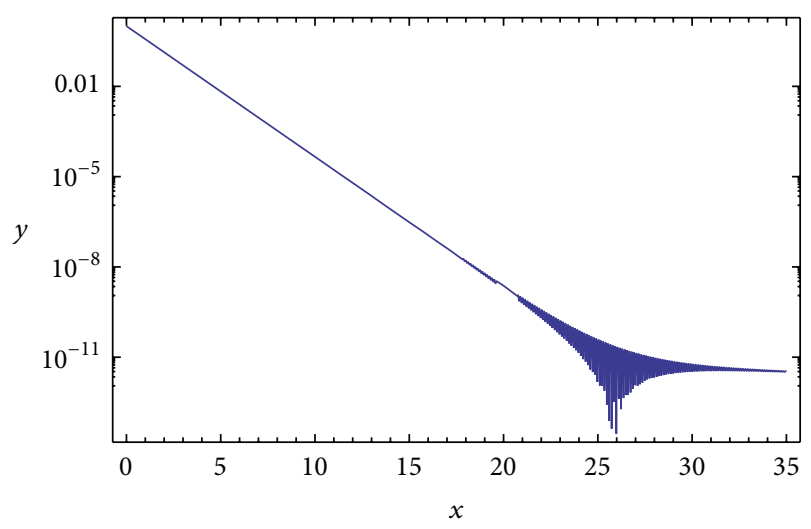

Figure 1: Decay curve obtained for $\omega(m)$ given by (6). Axes: $y=$ $\mathscr{P}_{0}(t)$, the logarithmic scale; $x=t / \tau_{0}$ (time is measured in lifetimes).

because, from the normalization condition $\int_{\mu_{0}}^{\infty}|c(m)|^{2} d m \equiv$ $\int_{\mu_{0}}^{\infty} \omega(m) d m=1$, it follows that $\omega(m)$ is an absolutely integrable function (note that this approach is also applicable in Quantum Field Theory models [24, 25]). The typical form of the survival probability $\mathscr{P}_{0}(t)$ is presented in Figure 1, where the calculations were performed for $\omega(m)$ having the Breit-Wigner form:

$$
\omega_{\mathrm{BW}}(m) \equiv \frac{N}{2 \pi} \Theta\left(m-\mu_{0}\right) \frac{\Gamma_{\phi}^{0}}{\left(m-M_{\phi}^{0}\right)^{2}+\left(\Gamma_{\phi}^{0} / 2\right)^{2}},
$$

assuming for simplicity that $s_{0}=\left(M_{\phi}^{0}-\mu_{0}\right) / \Gamma_{\phi}^{0}=50$. Here, $\Theta(m)$ is a step function: consider $\Theta(m)=0$ for $m \leq 0$ and $\Theta(m)=1$ for $m>0$.

Note that

$$
H|\phi\rangle \equiv \int_{\mu_{0}}^{\infty} m c(m)|m\rangle d m,
$$

which means that the vector $|\phi\rangle$ corresponding to an unstable state is not the eigenvector for the Hamiltonian $H$. In other words, in the rest frame considered, there does not exist any number; let us denote it by $m_{\phi}^{0}$, such that it would be $H|\phi\rangle=$ $m_{\phi}^{0}|\phi\rangle$. This means that the mass (i.e., the rest mass $m_{\phi}^{0}$ ) of the unstable quantum system described by the vector $|\phi\rangle$ is not defined. What is more, in such a case, the mass of this system cannot be constant in time in the state considered. Simply, the mass of the unstable system cannot take the exact constant value in the state $|\phi\rangle$; otherwise, it would not be any decay; that is, it would be $\mathscr{P}_{0}(t) \equiv|\langle\phi|\exp [-i t H]| \phi\rangle|^{2}=1$, for all $t$. In general, such quantum systems are characterized by the time independent mass (energy) distribution density $\omega(m)$, that is, by the modulus of the expansion coefficient $c(m)$, but not by the exact value of the mass. In this case, instead of the mass, the average mass, $\left\langle m_{\phi}\right\rangle$, of the unstable system can be determined knowing $\omega(m)$ or the instantaneous mass of this system [23, 26-28]. The average mass is defined by means of the standard formula: $\left\langle m_{\phi}\right\rangle=\int_{\mu_{0}}^{\infty} m \omega(m) d m$. The instantaneous mass $m_{\phi}^{0}(t)$ (energy) can be found using 
the exact effective Hamiltonian $h_{\phi}(t)$ governing the time evolution in the subspace of states spanned by the vector $|\phi\rangle$ :

$$
\begin{aligned}
h_{\phi}(t) & =\frac{i}{a(t)} \frac{\partial a(t)}{\partial t}, \\
& \equiv \frac{\langle\phi|H| \phi ; t\rangle}{\langle\phi \mid \phi ; t\rangle},
\end{aligned}
$$

which results from the Schrödinger equation when one looks for the exact evolution equation for the mentioned subspace of states (for details, see [23, 26-29]). Within the assumed system of units, the instantaneous mass (energy) of the unstable quantum system in the rest reference frame is the real part of $h_{\phi}(t)$ :

$$
m_{\phi}^{0}(t)=\Re\left[h_{\phi}(t)\right],
$$

and $\Gamma_{\phi}(t)=-2 \mathfrak{I}\left[h_{\phi}(t)\right]$ is the instantaneous decay rate.

Using relation (9), one can find some general properties of $h_{\phi}(t)$ and $m_{\phi}^{0}(t)$. Indeed, if to rewrite the numerator of the right-hand side of (9) as follows,

$$
\langle\phi|H| \phi ; t\rangle \equiv\langle\phi|H| \phi\rangle a(t)+\langle\phi|H| \phi ; t\rangle_{\perp},
$$

where $|\phi ; t\rangle_{\perp}=Q|\phi ; t\rangle, Q=\mathbb{Q}-P$ is the projector onto the subspace od decay products, $P=|\phi\rangle\langle\phi|$, and $\langle\phi \mid \phi ; t\rangle_{\perp}=0$, then one can see that there is a permanent contribution of decay products described by $|\phi ; t\rangle_{\perp}$ to the instantaneous mass (energy) of the unstable state considered. The intensity of this contribution depends on time $t$. Using (9) and (11), one finds that

$$
h_{\phi}(t)=\langle\phi|H| \phi\rangle+\frac{\langle\phi|H| \phi ; t\rangle_{\perp}}{a(t)} .
$$

From this relation, one can see that $h_{\phi}(0)=\langle\phi|H| \phi\rangle$ if the matrix elements $\langle\phi|H| \phi\rangle$ exist. It is because $|\phi(t=0)\rangle_{\perp}=0$ and $a(t=0)=1$. Now, let us assume that $\langle\phi|H| \phi\rangle$ exists and $i(\partial a(t) / \partial t) \equiv\langle\phi|H| \phi ; t\rangle$ is a continuous function of time $t$ for $0 \leq t<\infty$. If these assumptions are satisfied, then $h_{\phi}(t)$ is a continuous function of time $t$ for $0 \leq t<\infty$ and $h_{\phi}(0)=\langle\phi|H| \phi\rangle$ exists. Now, if to assume that for $0 \leq$ $t_{1} \neq t_{2}$ there is $\mathfrak{R}\left[h_{\phi}(0)\right]=\mathfrak{R}\left[h_{\phi}\left(t_{1}\right)\right]=\mathfrak{R}\left[h_{\phi}\left(t_{2}\right)\right]=$ const, then from the continuity of $h_{\phi}(t)$ it immediately follows that there should be $\mathfrak{R}\left[h_{\phi}(t)\right]=h_{\phi}(0) \equiv\langle\phi|H| \phi\rangle=$ const for any $t \geq 0$. Unfortunately, such an observation contradicts implications of (12): from this relation, it follows that $\mathfrak{R}\left[\langle\phi|H| \phi ; t\rangle_{\perp} / a(t)\right] \neq 0$ for $t>0$ and thus $\mathfrak{R}\left[h_{\phi}(t>\right.$ $0)] \neq\langle\phi|H| \phi\rangle \equiv \Re\left[h_{\phi}(0)\right]$ which shows that $m_{\phi}^{0}(t) \equiv$ $\Re\left[h_{\phi}(t)\right]$ cannot be constant in time. Results of numerical calculations presented in Figure 2 (or those one can find in [28]) confirm this conclusion.

In the general case, the mass (energy) distribution function $\omega(E)$ has properties similar to the scattering amplitude; that is, it can be decomposed into a threshold factor, a polefunction $P(m)$ with a simple pole at $m=M_{\phi}^{0}-(i / 2) \Gamma_{\phi}^{0}$ (often modeled by a Breit-Wigner), and a smooth form factor $F(m)$. So there is (see, e.g., [18])

$$
\omega(m)=\Theta\left(m-\mu_{0}\right)\left(m-\mu_{0}\right)^{\lambda+l} P(m) F(m),
$$


Figure 2: A typical form of the instantaneous mass $m_{\phi}(t)$ as a function of time obtained for $\omega_{\mathrm{BW}}(m)$. Axes: $y=\kappa(t)$, where $\kappa(t)$ is defined by (16); $x=t / \tau_{\phi}$ : time is measured in lifetimes. The horizontal dashed line represents the value of $m_{\phi}^{0}(t)=M_{\phi}^{0}$.

where $l$ is the angular momentum; $1>\lambda \geq 0$. In such a case,

$$
h_{\phi}^{0}(t) \simeq M_{\phi}^{0}-\frac{i}{2} \Gamma_{\phi}^{0}, \quad\left(t \sim \tau_{\phi}\right)
$$

at canonical decay times, that is, when the survival probability has the exponential form (here, $\tau_{\phi}$ is the lifetime), and

$$
\begin{aligned}
m_{\phi}^{0}(t)=\Re\left[h_{\phi}(t)\right] \simeq M_{\phi}^{0}=\left\langle m_{\phi}\right\rangle+\Delta m_{\phi}^{0}, & \\
& \left(t \sim \tau_{\phi}\right),
\end{aligned}
$$


at these times to a good accuracy (see [26, 27, 29]). The parameters $M_{\phi}^{0}$ and $\Gamma_{\phi}^{0}$ are the quantities that are measured in decay and scattering experiments. If the state vector $|\phi\rangle=$ $\left|\phi_{\alpha}\right\rangle$ is an eigenvector for $H$ corresponding to the eigenvalue $m_{\alpha}$, then there is $h_{\phi_{\alpha}}(t) \equiv m_{\alpha}$. Beyond the canonical decay times, $m_{\phi}^{0}(t)$ differs from $M_{\phi}^{0}$ significantly (for details, see [26-28]). At canonical decay times, values of $m_{\phi}^{0}(t)$ fluctuate (faster or slower) around $M_{\phi}^{0}$. One can see a typical behavior of $m_{\phi}^{0}(t)$ in Figure 2, where the function,

$$
\kappa(t)=\frac{m_{\phi}^{0}(t)-\mu_{0}}{M_{\phi}^{0}-\mu_{0}},
$$

is presented. These results were obtained numerically for the Breit-Wigner mass (energy) distribution function $\omega(m)=$ $\omega_{\mathrm{BW}}(m)$ and for $s_{0}=50$. From Figure 2 , one can see that fluctuations of $m_{\phi}^{0}(t)$ take place at all stages of the time evolution of the quantum unstable system. At times of order of the lifetime, $t \sim \tau_{\phi}$, and at shorter times, the amplitude of these fluctuations is so small that their impact on results of the mass (energy) measurements can be neglected (see (15)). With increasing time, their amplitude grows up to the maximal values, which take place at the transition times, that is, when the late time nonexponential deviations of the survival probability, $\mathscr{P}_{0}(t)$, begin to dominate. Thus, with the increasing time, for $t>\tau_{\phi}$, the impact of these fluctuations on behavior of the quantum unstable systems increases.

Now, let us consider the case when the unstable quantum system is moving with a velocity $\vec{v}$ relative to reference frame $\mathcal{O}$. It is obvious that an unstable quantum system moving with the relativistic velocity does not turn into a classical system but still subjects to the laws of quantum physics. So when one searches for properties of such systems, the implications following from rules of the quantum theory are decisive. Let us assume that this quantum object is moving freely with the constant velocity $\vec{v}$ :

$$
\vec{v}=\text { const, }
$$

and let us admit that $\vec{v}$ is so large that the relativistic effects can take place. The energy $E_{\phi}$ of the quantum unstable system described in the rest frame by vector $|\phi\rangle$ and moving with the constant velocity $\vec{v}$ can be expressed within the system of units used as follows:

$$
E_{\phi}=m_{\phi}^{0} \gamma
$$

where $m_{\phi}^{0}$ is the mass parameter (i.e., the rest mass) and $\gamma=$ const. Thus, knowing the energy $E_{\phi}$ and the velocity $\vec{v}$, that is, the Lorentz factor $\gamma$, one can determine the mass parameter $m_{\phi}^{0}$.

From the fundamental principles, it follows that the total energy of the freely moving objects, both quantum and classical, stable and unstable, must be conserved. This means that if an experiment indicates the energy, $E$, of such an object to be equal to $E\left(t_{1}\right)=E_{\phi}$ at an instant $t_{1}$, then at any instant $t_{2}>t_{1}$ there must be $E\left(t_{2}\right)=E\left(t_{1}\right) \equiv E_{\phi}$. Now, if the energy,
$E$, of the moving quantum unstable system is conserved, $E=$ $E_{\phi}=$ const, then from the assumption it trivially results that there must be

$$
m_{\phi}^{0}=\frac{E_{\phi}}{\gamma} \equiv \text { const. }
$$

This observation concerns also the instantaneous mass $m_{\phi}^{0}(t)$ : if it was $E_{\phi}=E_{\phi}(t)$, it would be $E_{\phi}(t) / \gamma=m_{\phi}^{0}(t)$. The conservation of the energy means that at any instant of time the energy has the same value, so there must be $E_{\phi}(t) \equiv E_{\phi}=$ const. Therefore, if the energy is conserved and assumption (17) holds, then there must be $m_{\phi}^{0}(t)=E_{\phi}(t) / \gamma \equiv E_{\phi} / \gamma=$ const.

On the basis of this analysis, one can conclude that the rest mass $m_{\phi}^{0}$ as well as the instantaneous mass $m_{\phi}^{0}(t)$ of the moving quantum unstable system are constant at all instants of time $t$. But, unfortunately, such a conclusion is in sharp contrast to the conclusion following from the relation (7) and its consequences. This means that one should consider the following possible situations: either (a) conclusions following from the quantum theoretical treatment of the problem are wrong (i.e., the quantum theory is wrong) or (b) the energy of moving quantum unstable systems is not conserved (i.e., the principle of the conservation of the energy does not apply to moving quantum unstable systems), or simply (c) the assumption (17) cannot be realized in the case of moving quantum unstable systems. The probability that situations (a) or (b) occur is rather negligible small. So the only reasonable conclusion is that case (c) takes place.

This situation has a simple explanation. Namely, despite the conclusions resulting from relation (7) in experiments with unstable particles, one observes them as massive objects. This is not in contradiction to the implications of relation (7). The conclusion that the mass of the unstable quantum system (i.e., the rest mass of such a system) cannot be defined and constant in time means that in the case of such system only the instantaneous mass varying in time can be considered: it can only be $m_{\phi}^{0} \equiv m_{\phi}^{0}(t)$.

So in the case of the moving quantum unstable system, the principle of the conservation of the energy takes the following form:

$$
E_{\phi}=m_{\phi}^{0}(t) \gamma(\vec{v}(t))=\text { const. }
$$

Thus, the principle of conservation of energy forces the compensation of changes in the instantaneous mass $m_{\phi}^{0}(t)$ through appropriate changes in the velocity $\vec{v} \equiv \vec{v}(t)$ so that the product $m_{\phi}^{0}(t) \gamma(\vec{v}(t))$ was fixed and constant in time: for any times $t_{1}>t_{2}$ (in general, $t_{1} \neq t_{2}$ ), there must be $m_{\phi}^{0}\left(t_{1}\right) \gamma\left(\vec{v}\left(t_{1}\right)\right)=m_{\phi}^{0}\left(t_{2}\right) \gamma\left(\vec{v}\left(t_{2}\right)\right)=E_{\phi}$ (it is a pirouette like effect). In other words, the principle of the conservation of the energy does not allow any moving quantum unstable system to move with the velocity $\vec{v}$ constant in time.

\section{Concluding Remarks}

The above conclusions result from the basic principles of the quantum theory. Taking this into account, one should 
consider a possibility that, in the case of moving quantum unstable systems, assumption (17) may lead to the wrong conclusions. In general, the relation $\mathscr{P}_{v}(t)=\mathscr{P}_{0}(t / \gamma)$ can be considered only as the approximate one and it cannot pretend to be rigorous. So instead of using such a relation, one should rather look for an effective formula for the survival probability of the moving quantum unstable systems. Such effective formula could be obtained, for example, by replacing $\gamma=$ const in $\mathscr{P}_{0}(t / \gamma)$ by an effective Lorentz factor $\gamma_{\text {eff }}(t)=$ $\gamma(\vec{v}(t)) \neq$ const, which varies with the changes of velocity $\vec{v}(t)$. Similar analysis shows that the assumption $\vec{p}=$ const leads to the conclusion analogous to that resulting from the assumption $E_{\phi}=$ const that the velocity $\vec{v}$ of the moving quantum unstable systems cannot be constant in time. This is the consequence of the relativistic formula for the momentum $\vec{p}$.

From results presented in Figure 2, it is seen that, with increasing time, $t$, the amplitude of fluctuations of $m_{\phi}(t)$ grows. So according to (20), in order to compensate these growing fluctuations, the fluctuations of the velocity $\vec{v}(t)$ of the unstable system have to grow. This means that, with increasing time, $t$, (at $t>\tau_{\phi}$ ), deviations of the decay law of moving unstable system from the classical relation $\mathscr{P}_{0}(t / \gamma)$ should be more visible and should grow. This effect explains the results presented in [3] where with the increasing time the increasing difference between $\mathscr{P}_{0}(t / \gamma)$ and $\mathscr{P}_{p}(t)$ was indicated and analyzed.

One more remark is as follows. Let us denote by $\sigma^{\prime}$ the reference frame which moves together with the moving quantum unstable system considered and in which this system is in rest. This reference frame moves relative to $\hat{\sigma}$ with the velocity $\vec{v}$. The property that the velocity $\vec{v}$ of the moving quantum unstable system cannot be constant in time has an effect that $d \vec{v} / d t \neq 0$. Therefore, the rest reference frame $\mathcal{O}^{\prime}$ of such a system cannot be the inertial one. This observation means that there does not exist a Lorentz transformation describing a transition from the inertial rest reference frame $\mathcal{O}$ of the observer into the noninertial rest reference frame $\mathcal{O}^{\prime}$ of the moving quantum unstable system.

The last remark is as follows. It seems that the abovedescribed effect can be relatively easily verified experimentally. It is because the conclusion that the velocity $\vec{v}$ of the moving quantum unstable system must vary in time means that $d \vec{v} / d t \neq 0$. Therefore, the moving freely charged unstable particles (or neutral unstable particles with nonzero magnetic moment) should emit electromagnetic radiation of the very broad spectrum: from very small up to extremely large frequencies (see [28]). Thus, this effect can be verified by using currently carried out experiments, which use a beam of charged unstable particles (e.g., $\pi^{ \pm}$mesons or muons) or ions of radioactive elements moving along a straight line. A section of the track of these particles, where they are moving freely, should be surrounded by sensitive antennae connected to the receivers being able to register a broad spectrum of the electromagnetic radiation. Then, every signal coming from the beam registered by these receivers will be the proof that the above-described effect takes place.

\section{Conflict of Interests}

The author declares that there is no conflict of interests regarding the publication of this paper.

\section{Acknowledgments}

The author would like to thank E. V. Stefanovich for valuable comments. The work was supported by the Polish NCN Grant no. DEC-2013/09/B/ST2/03455.

\section{References}

[1] E. V. Stefanovich, "Quantum effects in relativistic decays," International Journal of Theoretical Physics, vol. 35, no. 12, pp. 2539-2554, 1996.

[2] M. Shirokov, "Decay law of moving unstable particle," International Journal of Theoretical Physics, vol. 43, no. 6, pp. 1541-1553, 2004.

[3] K. Urbanowski, "Decay law of relativistic particles: quantum theory meets special relativity," Physics Letters B, vol. 737, pp. 346-351, 2014.

[4] P. Exner, "Representations of the Poincaré group associated with unstable particles," Physical Review D, vol. 28, no. 10, pp. 26212627, 1983.

[5] M. I. Shirokov, "Evolution in time of moving unstable systems," Concepts of Physics, vol. 3, pp. 193-205, 2006.

[6] M. I. Shirokov, "Moving system with speeded-up evolution," Physics of Particles and Nuclei Letters, vol. 6, no. 1, pp. 14-17, 2009.

[7] J. Bailey, K. Borer, F. Combley et al., "Measurements of relativistic time dilatation for positive and negative muons in a circular orbit," Nature, vol. 268, no. 5618, pp. 301-305, 1977.

[8] D. H. Frisch and J. H. Smith, "Measurement of the relativistic time dilation using $\mu$-mesons," American Journal of Physics, vol. 31, pp. 342-355, 1963.

[9] F. Giacosa, "Decay law and time dilation," http://arxiv.org/abs/ 1512.00232.

[10] E. V. Stefanovich, "Relativistic quantum dynamics: a nontraditional perspective on space, time, particles, fields, and action-at-a-distance," http://arxiv.org/abs/physics/0504062.

[11] E. V. Stefanovich, "Violations of Einstein's time dilation formula in particle decays," http://arxiv.org/abs/physics/0603043.

[12] B. T. Shields, M. C. Morris, M. R. Ware, Q. Su, E. V. Stefanovich, and R. Grobe, "Time dilation in relativistic two-particle interactions," Physical Review A, vol. 82, no. 5, Article ID 052116, 2010.

[13] L. A. Khalfin, "Quantum theory of unstable particles and relativity," PDMI Preprint 6/1997, St. Petersburg Department of Steklov Mathematical Institute, St. Petersburg, Russia, 1997.

[14] N. S. Krylov and V. A. Fock, "O dvuh osnovnyh tolkovanijah sootnosenija neopredelenosti dla energii i vremeni," Zhurnal Eksperimental'noi i Teoreticheskoi Fiziki, vol. 17, pp. 93-107, 1947 (Russian).

[15] V. A. Fock, Fundamentals of Quantum Mechanics, Mir Publishers, Moscow, Russia, 1978.

[16] L. A. Khalfin, "K teorii raspada kvasistacionarnogo sostojanija," Zhurnal Eksperimental'noi i Teoreticheskoi Fiziki, vol. 33, pp. 1371-1382, 1957 (Russian).

[17] L. A. Khalfin, "Contribution to the decay theory of a quasistationary state," Soviet Physics-JETP, vol. 6, p. 1053, 1958. 
[18] L. Fonda, G. C. Ghirardi, and A. Rimini, "Decay theory of unstable quantum systems," Reports on Progress in Physics, vol. 41, no. 4, pp. 587-631, 1978.

[19] J. Martorell, J. G. Muga, and D. W. L. Sprung, "Quantum postexponential decay," in Time in Quantum Mechanics_Vol. 2, vol. 789 of Lecture Notes in Physics, pp. 239-275, Springer, Berlin, Germany, 2009.

[20] E. Torrontegui, J. G. Muga, J. Martorell, and D. W. L. Sprung, "Quantum decay at long times," Advances in Quantum Chemistry, vol. 60, pp. 485-535, 2010.

[21] N. G. Kelkar and M. Nowakowski, "No classical limit of quantum decay for broad states," Journal of Physics A, vol. 43, no. 9, Article ID 385308, 2010.

[22] L. M. Krauss and J. Dent, "Late time behavior of false vacuum decay: possible implications for cosmology and metastable inflating states," Physical Review Letters, vol. 100, no. 17, Article ID 171301, 4 pages, 2008.

[23] F. Giraldi, "Logarithmic decays of unstable states," The European Physical Journal D, vol. 69, article 5, 8 pages, 2015.

[24] F. Giacosa, "Non-exponential decay in quantum field theory and in quantum mechanics: the case of two (or more) decay channels," Foundations of Physics, vol. 42, no. 10, pp. 1262-1299, 2012.

[25] M. L. Goldberger and K. M. Watson, Collision Theory, Wiley, 1964.

[26] K. Urbanowski, "Long time properties of the evolution of an unstable state," Open Physics, vol. 7, no. 4, pp. 696-703, 2009.

[27] K. Urbanowski, "General properties of the evolution of unstable states at long times," European Physical Journal D, vol. 54, no. 1, pp. 25-29, 2009.

[28] K. Urbanowski and K. Raczyńska, "Possible emission of cosmic $X$ - and $\gamma$-rays by unstable particles at late times," Physics Letters $B$, vol. 731, pp. 236-241, 2014.

[29] K. Urbanowski, "Early-time properties of quantum evolution," Physical Review A, vol. 50, pp. 2847-2853, 1994. 

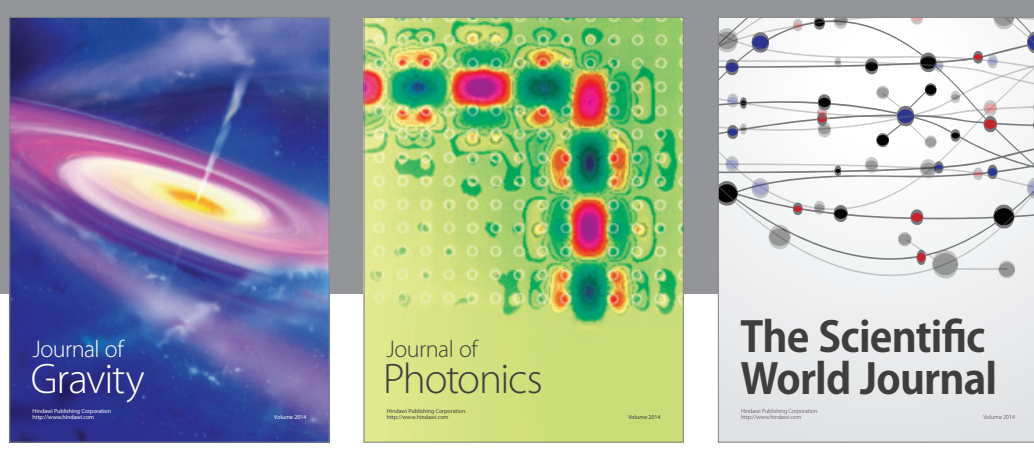

The Scientific World Journal
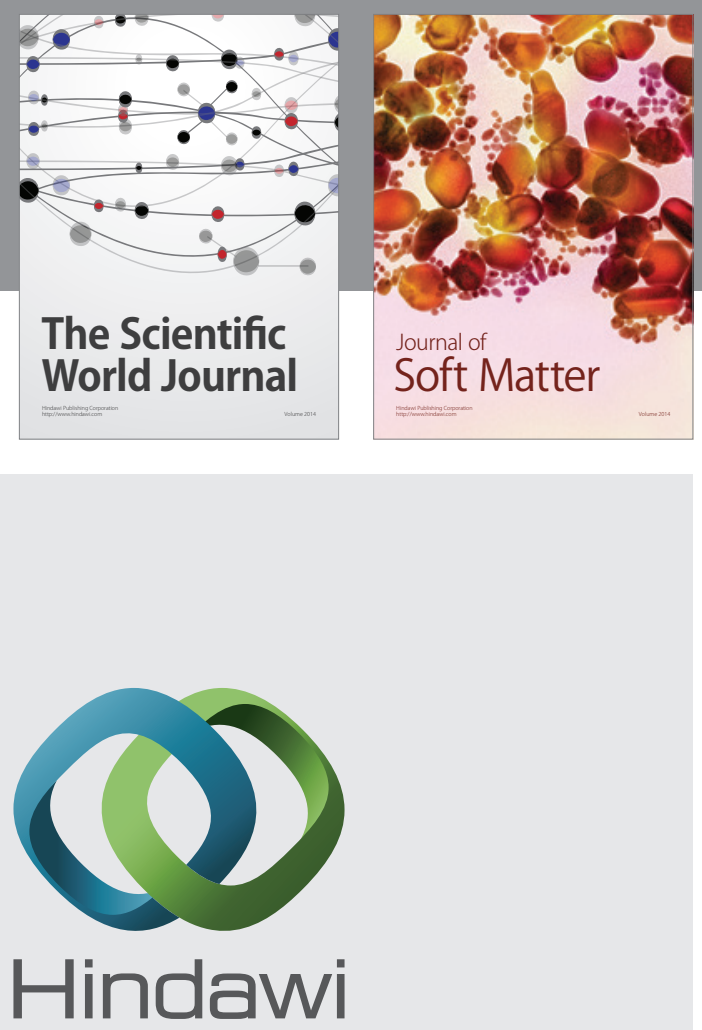

Submit your manuscripts at

http://www.hindawi.com

nternational Journal of

Statistical Mechanics
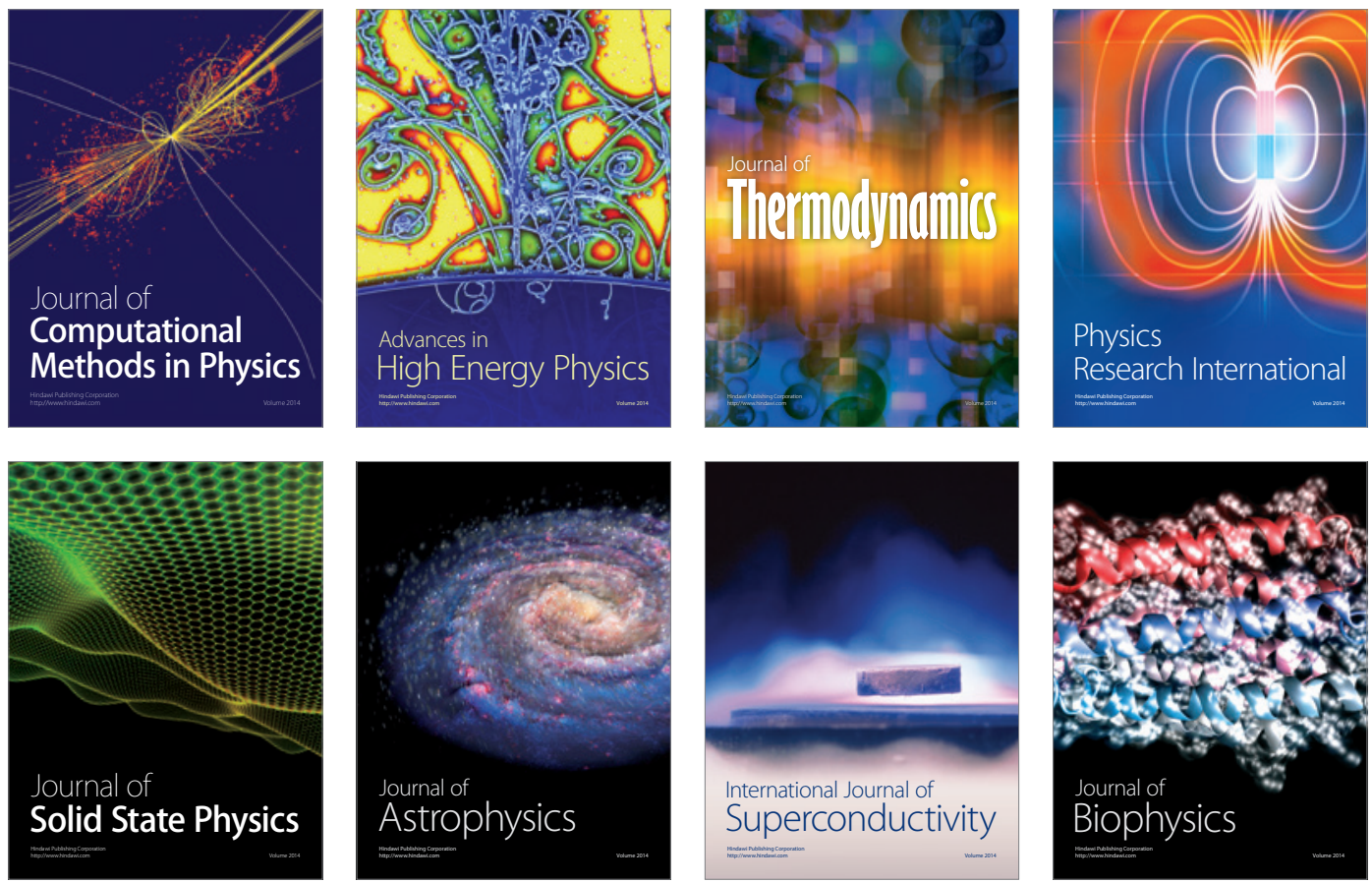
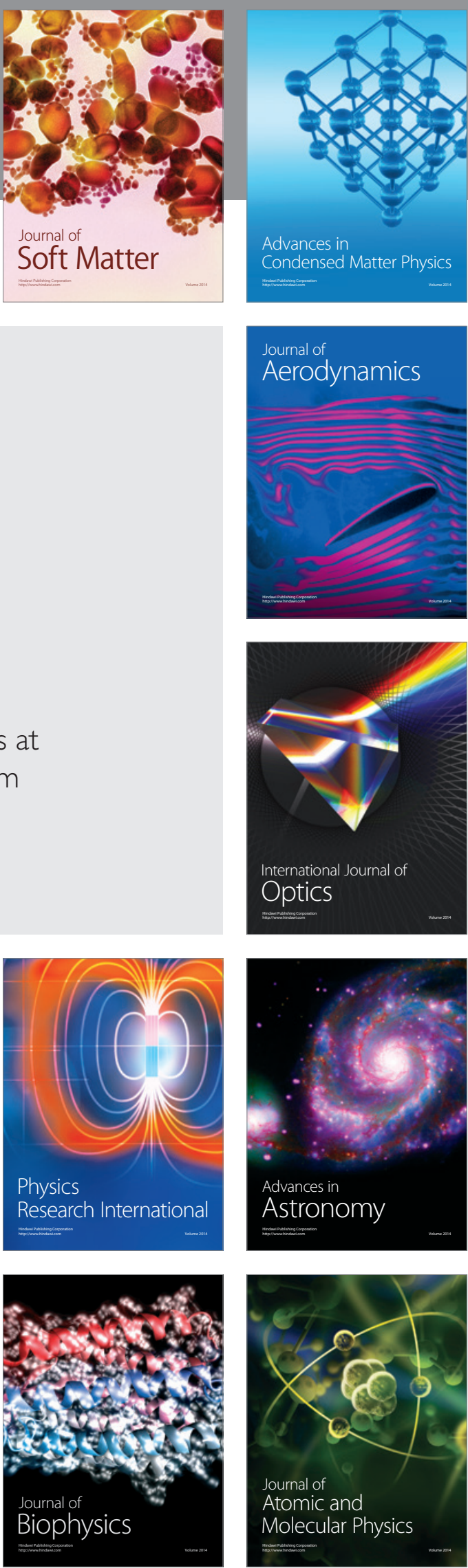